\title{
Comunicación online en el sector salud. Valor de la infografía
}

\author{
Por Toni González-Pacanowski y Pablo Medina
}

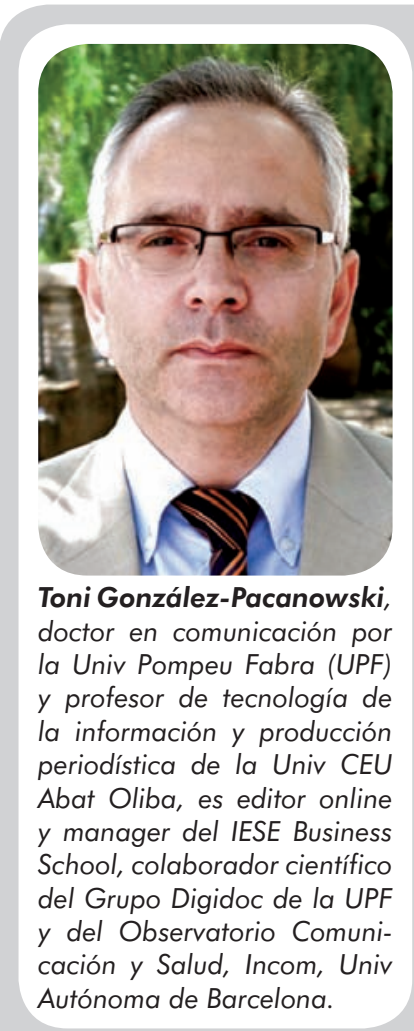

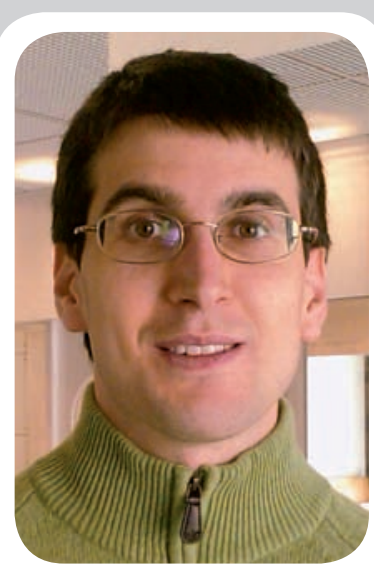

Pablo Medina-Aguerrebere es licenciado en publicidad y relaciones públicas por la Universidad de Navarra, y ha trabajado cuatro años en el sector de la comunicación y salud. En la actualidad prepara una tesis doctoral sobre dicho campo en la Universidad de Navarra.
Resumen: El gran interés que genera la salud entre la población ha llevado a varias instituciones españolas a potenciar el valor comunicacional de newsletters, foros online de pacientes, dípticos, vídeos online, teléfono móvil e infografía, entre otros. El objetivo de educar al paciente y de diferenciarse estratégicamente gracias al uso de la comunicación encuentra en la tecnología un aliado estratégico que supone muchos beneficios para el público (inmediatez, interactividad) y para la organización (imagen de marca, optimización del tiempo). Una de las iniciativas más representativas de esta corriente es la creación de infografías sobre temas de salud que, respetando el rigor científico, informativo, didáctico y ético, se difunden a través de distintos medios.

Palabras clave: Infografía, Salud, Comunicación, Educación, Divulgación, Tecnología.

\section{Title: Online communication in the health field. Value of the infography}

Abstract: The great interest that health generates in people has led to several Spanish institutions of the health sector to improve the communication value of newsletters, online patients forums, brochures, online videos, mobile phone and infography, among others. The aim to educate the patients and differing themselves strategically from other institutions finds in the technology a strategic partner that affords many benefits to the target audience (immediacy, interactivity) and to the organization (brand image, time optimization). One of the most representative initiatives of this trend is the creation of infographies about health matters that, respecting the scientific, informative, didactic and ethical rigour, are spread throughout different media.

Keywords: Infograhpy, Health, Communication, Education, Dissemination, Information technologies.

González-Pacanowski, Toni; Medina, Pablo. “Valor comunicacional de la infografía”. El profesional de la información, 2009, julio-agosto, v. 18, n. 4, pp. 413-420.

DOI: 10.3145/epi.2009.jul.08

\section{Introducción}

UNA DE LAS CORRIENTES MÁS INTERESANTES que tienen lugar en el sector de la salud en estos últimos años es la incorporación de la comunicación como sistema de diferenciación estratégica.

Aseguradoras, empresas farmacéuticas e incluso hospitales han apostado de forma genérica por el uso de la comunicación, especialmente la interactiva, la cual hace posible transmitir a pacientes, empleados y al resto de stakeholders de la organización distintas infor- maciones sobre temas tan diversos como los hábitos de vida saludables, los tratamientos médicos o las consecuencias para el sector sanitario de los cambios que tienen lugar en la sociedad, como por ejemplo el envejecimiento de la población, o la falta de profesionales médicos.

Este interés creciente por el campo de la comunicación ha llevado a los diferentes actores sanitarios a confeccionar distintas aplicaciones de información online como newsletters, foros de pacientes, dípticos online, vídeos e incluso sistemas de comunicación con el paciente a través del teléfono móvil. Entre estas iniciativas destaca el caso de la infografía, la cual tiene un gran valor comunicacional ya que, por un lado, transmite la información de forma gráfica y clara, lo que facilita su comprensión por parte del destinatario, y por otro lado, consigue mantener el rigor científico al que deben someterse todas las informaciones emitidas por los actores del sector salud.

Para clarificar la importancia comunicacional de la información online en el sector salud, estructuramos este trabajo en tres partes: 
- contexto de la comunicación y la salud,

- dispositivos de información online, $\mathrm{y}$

- uso de la infografía por parte de la Clínica Universidad de Navarra.

\section{Comunicación y salud}

La salud es uno de los temas que más interesa a la población española, lo cual ha llevado a los medios de comunicación a dar más cabida a este tema. Así, por ejemplo, los periódicos El país, El mundo, ABC y La vanguardia han pasado de publicar 4.079 artículos dedicados a la salud en 1997, a 9.247 artículos en $2007^{1}$.

\section{"En diez años se han duplicado los artículos sobre salud en la prensa"}

Los medios de comunicación y las organizaciones del sector sanitario no son los únicos en potenciar la comunicación en salud, ya que, diversas instituciones como agencias de viajes ${ }^{2}$, empresas de alimentación ${ }^{3}$ e incluso los departamentos de recursos humanos de las empresas $^{4}$ han apostado por integrar en sus unidades de negocio y en su estrategia de comunicación un componente de salud. Sin embargo la comunicación de salud no debe convertirse en un tema desvirtuado por actores que no están directamente relacionados con el sector salud, aunque hay que aceptar que la comunicación de salud es un fenómeno vital, que está directamente relacionado con las experiencias humanas y con las aspiraciones del hombre (Brashers; Babrow, 1996).

Una de sus peculiaridades más importantes es la necesidad de divulgar conocimientos científicos de forma rigurosa a la vez que se educa a un público general. En esta línea Martínez-Solana (2006), refiriéndose al caso de los comunicadores de salud, considera que la profesión de éstos tiene una doble faceta de divulgación científica, por un lado, y de interpretación sobre dichos asuntos, por otro. En cualquier caso la educación de la población en hábitos de vida saludables es un tema que está relacionado directamente con el concepto de motivación, la cual, según Suárez-Riveiro y Fernández-Suárez (2004), tiene un papel fundamental en el aprendizaje, especialmente cuando ayuda a establecer metas cercanas.

Por otro lado, el uso de las nuevas tecnologías es determinante, especialmente internet (foros, blogs, consultorios online y webs), que según Sierra-Caballero (2002), tiene un poder muy importante como método educativo. En esta línea, merecen especial atención por el valor educativo y comunicacional que poseen, los servicios de documentación personalizados, tanto los destinados a los profesionales de la salud como los destinados al público general ${ }^{5}$. Por otro lado, Turner (2003) también considera que la evolución de internet ha permitido que la telemedicina goce de un mayor impulso gracias, entre otras cosas, a las videoconferencias.

En esta línea, dos de las iniciativas que ayudan los profesionales de la salud -pero también al público general-, y que son posibles gracias a internet, son los servicios de segunda opinión médica y los portales de autogestión de la salud ${ }^{6}$. En cualquier caso, no toda la gente que accede en internet a información sobre salud la entiende de forma perfecta (Wright, Sparks; O'Hair, 2008), lo cual puede dar lugar a la cibercondría ${ }^{7}$, sin embargo, a pesar de todo internet, al igual que el resto de nuevos medios tecnológicos, es un garante de la democratización de la cultura (Sierra-Caballero,
2002), y, en el caso del sector hospitalario, un aliado estratégico muy importante ya que le facilita al médico optimizar su tiempo a través de indicar al paciente las páginas web donde hay cierta información que éste pide y que en el tiempo de una consulta no da tiempo a transmitir (Fisac, 2006).

\section{Iniciativas de información online en salud}

\subsection{Newsletters}

Las newsletters ayuda a la organización a establecer contactos duraderos en el tiempo con sus clientes (Jackson, 2004). En el sector salud esto es muy apreciado, ya que, por un lado, puede dar lugar a la creación de comunidades de usuarios, y por otro, facilita las labores de educación en salud. Además, si se usan junto con otros medios online tienen ayudan en la generación de campañas virales entre los destinatarios (Gosselin, 2008). Por otra parte, las newsletters también tienen un valor comunicacional dentro de la organización para ayudar a los directivos a gestionar mejor la información con el resto de empleados. Esto es algo primordial ya que la confianza que los directivos de una organización generan en el resto de empleados está influenciada por la calidad de la información que ellos transmiten (Byrne, 2006).

\subsection{Foros de pacientes}

En el contexto actual en el que los numerosos avances que tienen lugar en la medicina vuelven a ésta más compleja, hay un aumento de la información sobre salud disponible en internet y un mayor valor otorgado a las elecciones hechas por el paciente (Reid, 2008). Varias organizaciones del sector salud han creado foros online de pacientes en los que éstos reciben consejos y formación por parte de profesionales de la salud. El estado de ansiedad con el que 
muchos pacientes acuden a la consulta les impide expresarse bien verbalmente (Bensing, Verheul; Dulmen, 2008), de ahí la utilidad de que puedan acceder a un foro online para informarse sobre los temas que les preocupan antes de ir a la consulta, estando luego en mejores condiciones de comprensión y aprendizaje. A pesar de todo no hay que olvidar que la información de la que dispone el paciente no sólo proviene de internet, sino también de su propia experiencia, de las experiencias contadas por sus amigos y de la información vista en los medios de comunicación (Gwyn, 2002). Por eso los foros de pacientes online deben tener un enfoque global en el que se dé cabida a la voz del médico, pero también a la de otros pacientes. De hecho los pacientes que han sufrido experiencias y patologías similares pueden influenciar positivamente en las percepciones de otros pacientes (Wright, Sparks; O'Hair, 2008).

\section{"Internet ayuda al médico a optimizar su tiempo de trabajo"}

\subsection{Dípticos y vídeos online}

Teniendo en cuenta que los dípticos influyen directamente en la creación de relaciones duraderas con el cliente (Long, McMellon, Clark; Shiffman, 2006), y que además resultan muy útiles para los pacientes, ya que los pueden examinar fuera del entorno estresante de la consulta (Berry, 2006), es comprensible que algunas organizaciones, especialmente los hospitales, apuesten por ellos. Además, la creación de versiones online de dichos dípticos aporta mucho valor comunicacional porque son usados por los pacientes para tomar decisiones sobre los tratamientos médi- $\cos$ (Spyt, Watt, Boehm; Stafford, 2002).

Varios autores (Houston; Allison, 2002) han estudiando las ventajas y valores de los vídeos creados con la colaboración de los profesionales de la salud, dirigidos al público. También se han analizado los vídeos online al servicio de los propios profesionales médicos (Baldwin, Clarke, Eldabi y Jones, 2002).

\subsection{Teléfono móvil}

Se trata de un soporte cada vez más usado en el ámbito sanitario, especialmente en el contexto hospitalario, donde el teléfono permite gestionar las citas con el médico ${ }^{8}$, o simplemente mejora las relaciones comunicativas mantenidas entre el personal médico y el paciente ${ }^{9}$. Sin embargo, el uso del teléfono móvil para realizar acciones de comunicación y marketing exige previamente la aceptación y consentimiento por parte del usuario (Barnes; Scornavacca, 2004). En cualquier caso, el valor comunicacional del teléfono móvil en el sector sanitario queda demostrado debido a tres razones:

- factores que determinan el éxito de este soporte son la atención, la intención y el comportamiento del consumidor (Scharl, Dickinger; Murphy, 2005), y, en el sector de la salud, dichos factores pueden ser fácilmente trabajados por las instituciones participantes;

- el teléfono móvil es un medio que complementa y refuerza otros soportes de comunicación como la web (Reuther, 2008), la cual es muy potenciada por los actores del sector salud;

- el teléfono móvil se ha convertido en uno de los mecanismos más importantes que pueden utilizar las marcas para generar comunidades de usuarios (Perey, 2008), algo que cada vez es más común en el mundo de la salud y que se plas- ma, por ejemplo, en la constitución de asociaciones de pacientes.

\subsection{Infografía}

El carácter didáctico y educativo de la infografía la convierte en una de las actividades de información online más usadas en el sector salud. Teniendo en cuenta que las campañas de comunicación de salud pueden influenciar en el comportamiento sanitario de la población (Hornik, 2002), que la población está muy acostumbrada a la cultura de la imagen (Manovich, 2005), y que en la actualidad hay una fusión de soportes tecnológicos, parece claro que la difusión de infográficos por parte de las organizaciones implicadas en la salud es una de las estrategias más claras para lograr ese doble objetivo de divulgación científica rigurosa y de educación a la población.

En cualquier caso, la infografía de salud debe aportar un valor comunicacional a la institución, para lo cual es necesario respetar tres reglas. En primer lugar, hay que cumplir el rigor informativo, ya que al fin y al cabo, según ValeroSancho (2001), la infografía es una aportación informativa cuyo objetivo es facilitar la comprensión de acontecimientos. En segundo lugar, el infográfico debe respetar el rigor científico. Para ello, la colaboración entre el médico y el periodista a la hora de elaborar materiales de comunicación resulta muy útil (Fisac, 2006). Por último, el infografista debe ser consciente de la labor didáctica de su trabajo. En esta línea Franco-Álvarez (2005: 27) considera que la misión principal de la información didáctica es la transmisión de conocimientos con el objetivo de aumentar el nivel de formación del receptor. Por último a estas tres reglas es necesario añadir que el infografista también debe respetar la ética, la cual, debe estar presente en todas las actividades de comunicación y salud (Guttman, 2003). 


\section{El caso de la Clínica Universidad de Navarra}

Entre los diferentes actores de la comunicación y salud en España, merece especial atención la Clínica Universidad de Navarra, organización que entre sus distintas acciones de comunicación, dedica especial atención al uso de la infografía como medio de educación al paciente. Los infográficos son realizados para mostrar al paciente y a sus familiares cuál es el procedimiento de un tratamiento médico, cuáles son las consecuencias de una enfermedad o simplemente cómo es el funcionamiento de un equipo de radiodiagnóstico. Según Valero-Sancho (2002) la vista constituye una de las vías de entrada más importantes a través de las cuales el hombre accede al conocimiento, sin embargo, la vista también es vulnerable y puede sucumbir al engaño. Siguiendo con las palabras del autor, se puede decir que la infografía de salud tiene un potencial didáctico muy impor- tante, el cual debe ser combinado con una gran responsabilidad a la hora de crear los contenidos, algo que está muy presente en el trabajo infográfico realizado por la Clínica Universidad de Navarra.

Con el objetivo de revisar algunos conceptos infográficos, consideraremos tres aspectos:

- estructura del contenido,

- navegación, y

- valor educacional.

$\mathrm{Y}$ analizaremos tres de los infográficos realizados por la citada Clínica ${ }^{10}$.

Siguiendo la clasificación de Colle (2004), los tres infográficos reproducidos en este artículo pertenecen a la categoría de divulgación, ya que tienen por objetivo la transmisión de conocimientos científicos al público general ${ }^{11}$. Por eso, teniendo en cuenta la responsabilidad social que implica la transmisión de información sobre salud, una de las máximas de dichos infográficos es respetar los criterios de calidad marcados por Valero-Sancho (2001) y que se resumen en que:

- sean informativos, claros y comprensibles,

- respeten el aspecto estético y usen iconos,

- cuiden la tipografía y sean convenientes, y

- respeten la concordancia a nivel lingüístico y temporal.

En líneas generales se puede decir que dichos criterios de calidad son respetados por los tres infográficos ya que usan un lenguaje de divulgación, que respeta el rigor científico y que ayuda al público general comprender, en este caso, qué es el parkinson, el alzheimer o cómo funciona un microscopio fluorescente sin necesidad de tener conocimientos previos sobre el tema.

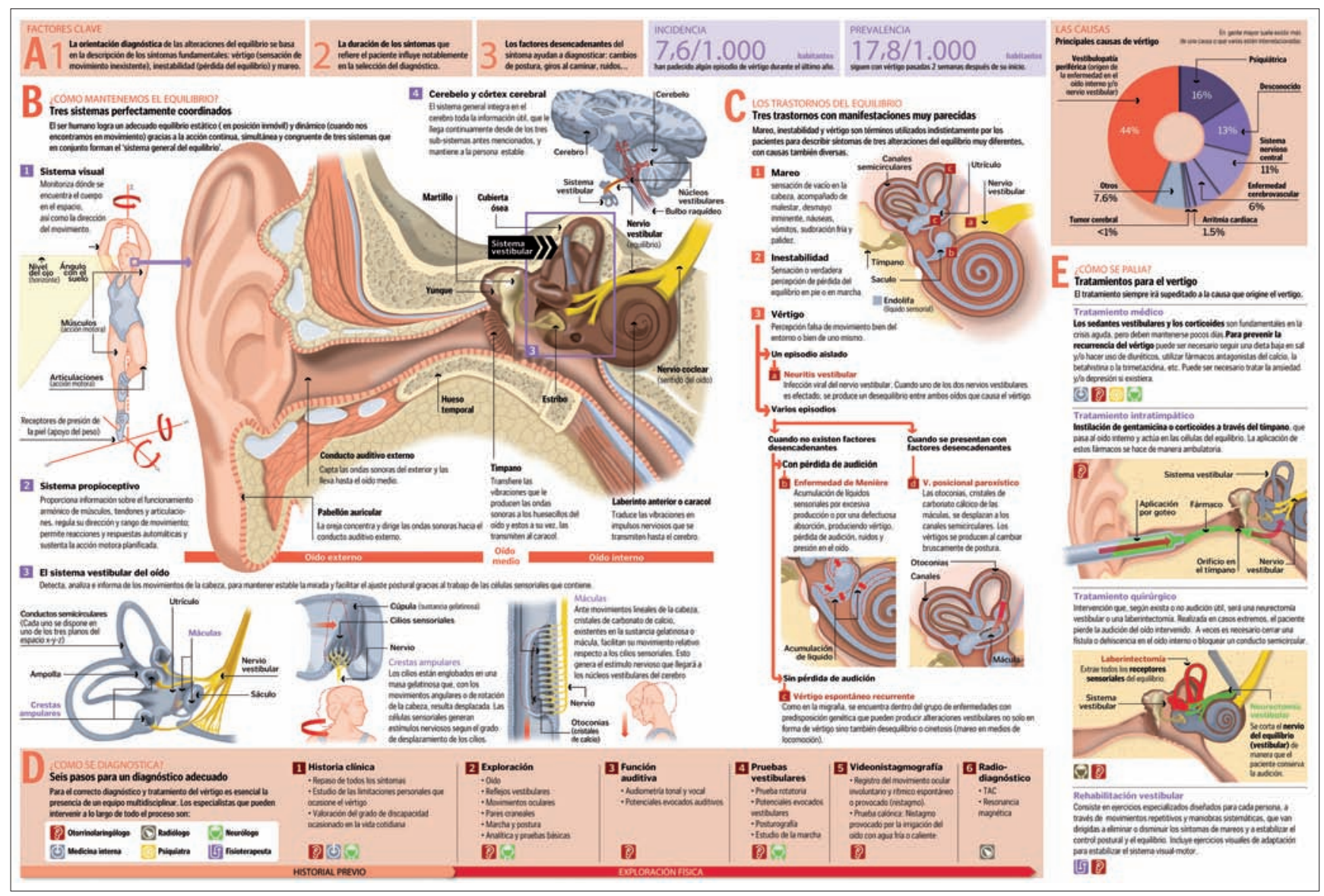

Figura 1. Trastornos del equilibrio 


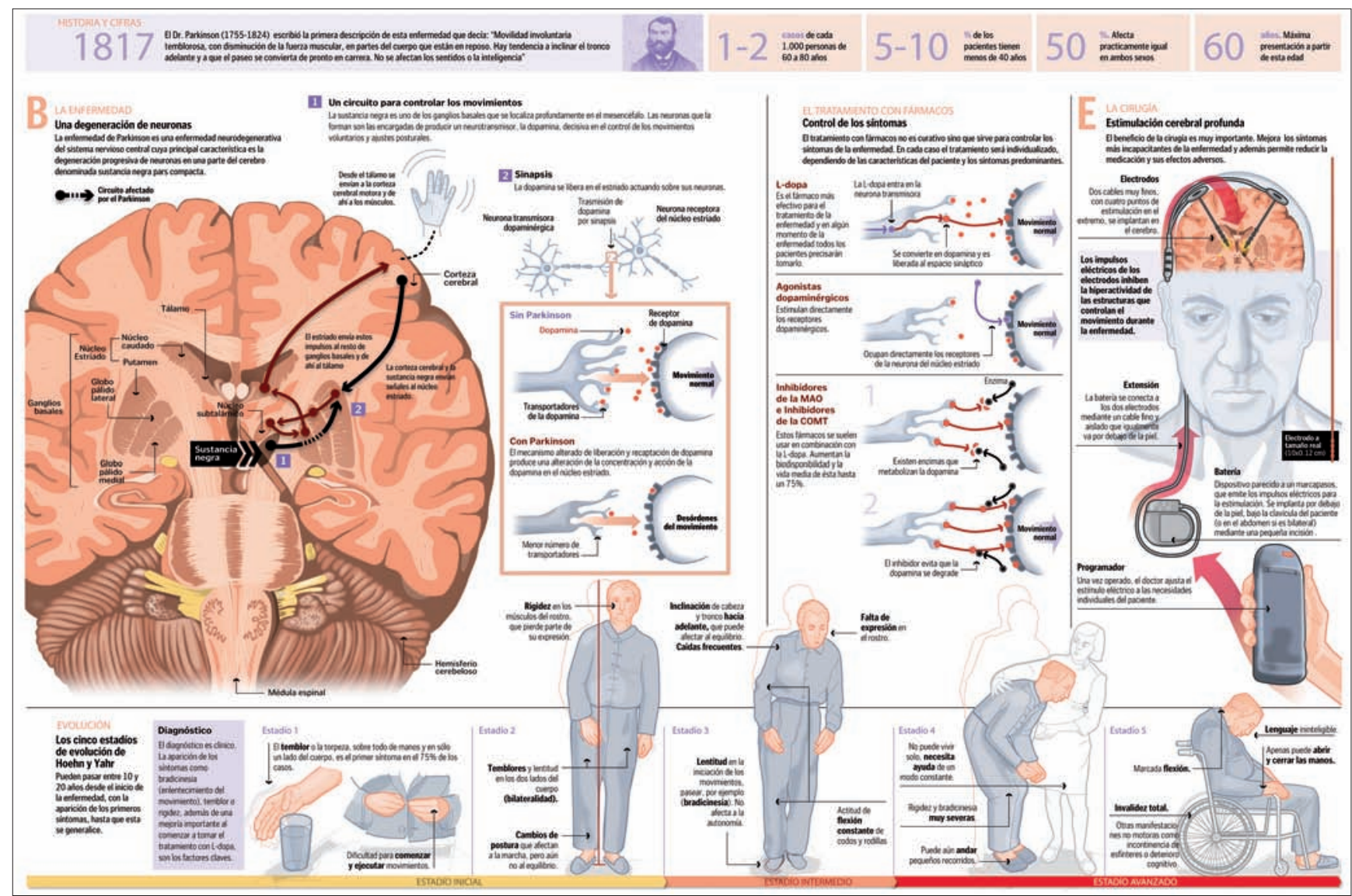

Figura 2. Parkinson

\section{Estructura del contenido}

Lo primero que hay que indicar es que los titulares son claros y breves (en el caso del tercer infográfico, por ejemplo, alguno de los titulares son: "Microscopio fluorescente", "Cabezal del microscopio", o "Fases de la operación"), lo cual ayuda a situar la información de forma rápida y clara.

El texto se ofrece en pequeñas píldoras de información que garantizan la comprensión del infográfico de forma global, aunque, también sería recomendable citar las fuentes usadas para poder ampliar información sobre el tema.

En cualquier caso, en los tres infográficos hay un buen equilibrio de texto e imagen.

Los infográficos de trastornos de equilibrio y de parkinson incluyen cierta sensación de movimiento, lo cual ayuda a crear la tridimensionalidad, que a su vez aumenta la capacidad de atracción. Además la gama de colores utilizados es reducida, es decir, no hay un uso abusivo de colores diferentes, lo cual los hace agradables y con buena visualidad. La búsqueda de la visualidad es muy importante en infografía ya que, en palabras de Valero-Sancho (2002), es la razón de ser de un infográfico, en el sentido de que éste es "visual" cuando gracias al mismo se puede explicar mejor una información. Sin embargo, estos infográficos no usan de forma clara una de las técnicas que, según Lallana (1999), resultan más interesantes que es la combinación de dibujo y fotografía.

\section{Navegación}

Los infográficos siguen el orden mental utilizado por el público (de izquierda a derecha, y de arriba abajo), señalando con letras o números el orden de lectura, y destacando en tamaño el elemento que tiene más valor informativo. Es decir, los tres infográficos siguen un orden temporal de los acontecimientos que tienen lugar y que conciernen a la patología (parkinson, trastornos del equilibrio) o al equipo de diagnóstico (microscopio fluorescente).

\section{Valor educacional}

A pesar de tratar temas complejos, los tres infográficos logran ser comprensibles por el público general gracias a su disposición, al lenguaje utilizado, la importancia dada a la imagen y el tono divulgativo. Por ello se puede apuntar que cumplen con la misión de educar al paciente, y que a su vez, respetan uno de los elementos que más se deben cuidar según Valero-Sancho (2000) y que es la concordancia con el idioma y la sintaxis, con el acontecimiento y con la información escrita que sirve de base.

\section{Conclusión}

El gran interés que la población tiene por los temas de salud repre- 


\section{MICROSCOPIO FLUORESCENTE}

El microscopio consigue hacer visibles los tumores cerebrales

para que el cirujano pueda extirparlos con una mayor precisión

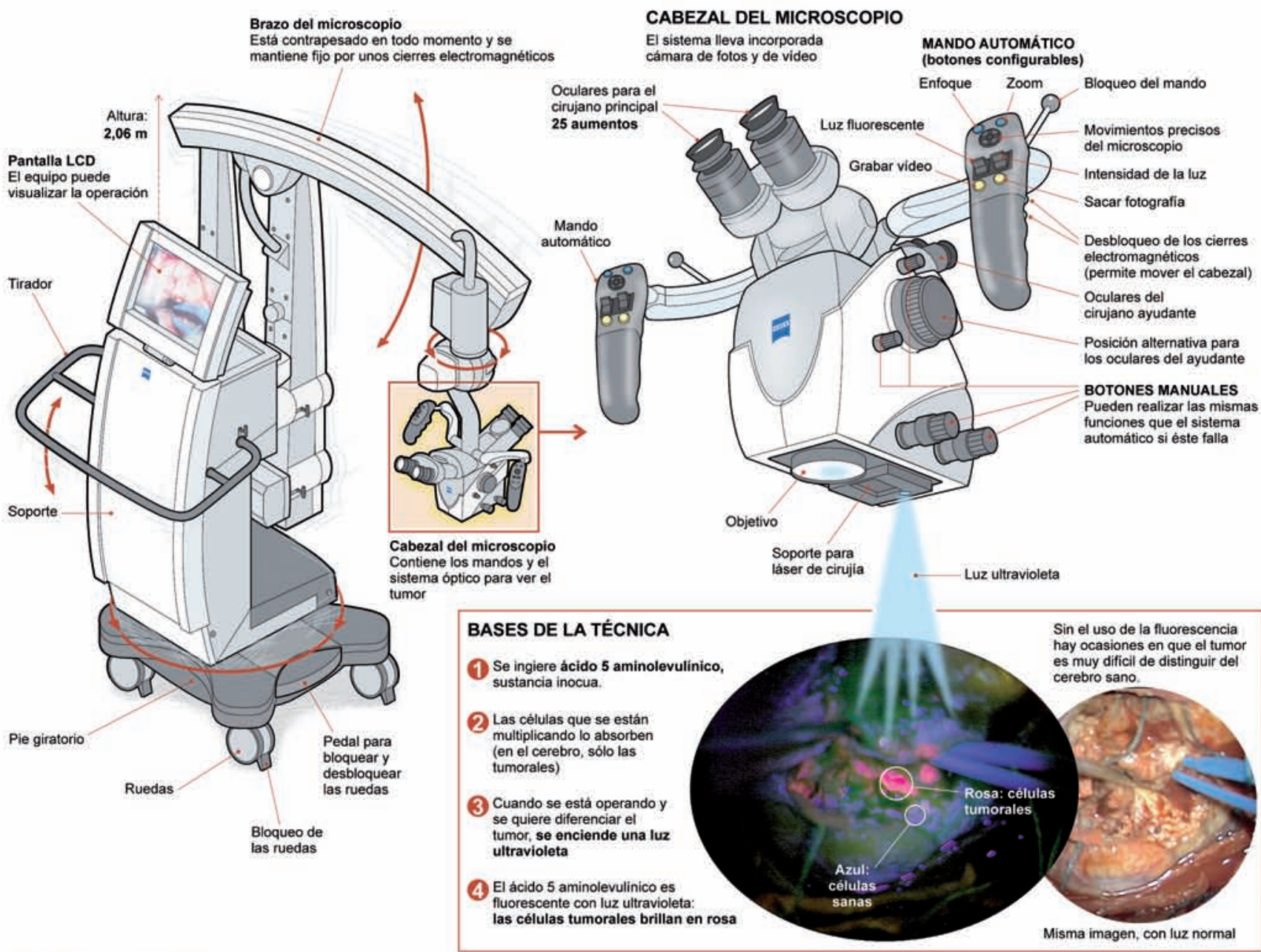

FASES DE LA OPERACIÓN

Se hace coincidir la operación con el momento

en el que el tumor es más fluorescente
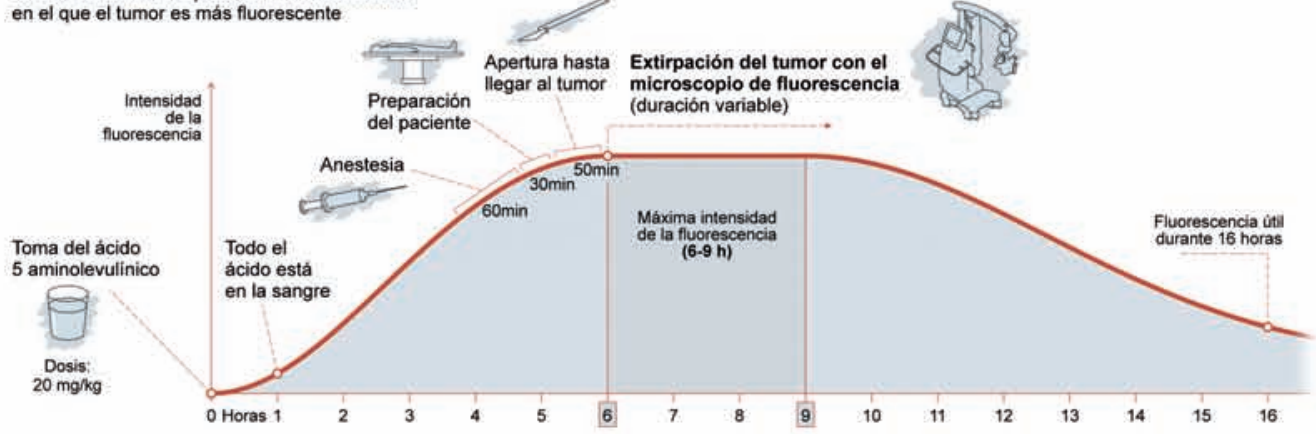

\section{LOS RESULTADOS}

Estudio en varios hospitales alemanes

En \% sobre el total de pacientes
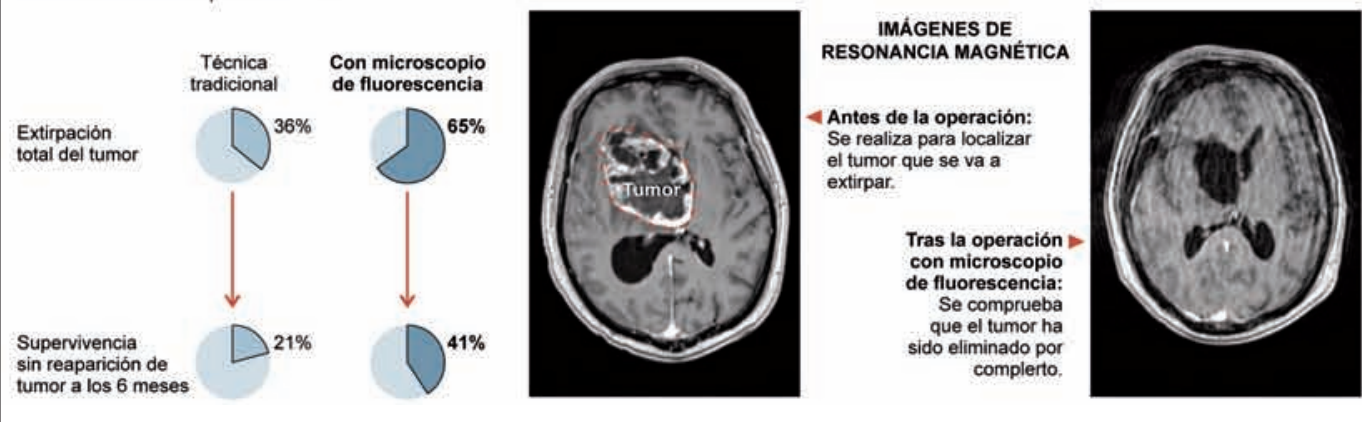

Figura 3. Microscopio fluorescente 
senta una oportunidad única para que los actores implicados directamente en ese área fomenten, gracias al uso de las nuevas herramientas tecnológicas, distintas iniciativas que tengan como objetivo la educación de la población en hábitos de salud. Este enfoque educacional resulta el más útil para que las distintas iniciativas de información online alcancen, por un lado, el mejor valor comunicacional posible, y por otro, para que la organización transmita hacia fuera una imagen de responsabilidad social.

\section{Notas}

1. JANO. La información de salud en la prensa general se ha duplicado en la última década. 13 de marzo de 2009.

http://www.jano.es/jano/actualidad/ultimas/ noticias/janoes/informacion/salud/prensa/ general/duplicado/ultima/decada/_f-11+iditem5897+idtabla-1

2. En España, en 2005 el volumen de negocio generado por el conjunto de establecimientos especializados en servicios de salud y belleza fue de 2.250 millones de euros, lo que representa un aumento del 32,5\% respecto al año anterior. $D B K$. Fuerte crecimiento del turismo de salud y belleza, julio de 2006.

http://www.dbk.es/esp/informaciongratuita/ datosPlantilla.cfm?iddatosSector $=3440$

3. Según el estudio «Hábitos de alimentación y salud» elaborado por el Instituto Omega 3 de la Fundación Puleva, el $42 \%$ de los españoles se preocupa más por la salud que hace diez años y el $47 \%$ considera que una alimentación sana es lo más importante para la salud.

Consumer Eroski. Los españoles se preocupan más por la salud y por una alimentación sana que hace 10 años, según un estudio, 7 nov 2001. http://www.consumer.es/web/es/salud/2001/ 11/07/47093.php

4. En España se puede citar el caso de Acciona, que propone a sus empleados cursos de alimentación sana; de Endesa, que tiene su propio equipo médico; de Iberdrola, que dispone de un gimnasio y de un fisioterapeuta; o el de Procter $\&$ Gamble, que propone a sus empleados programas para dejar de fumar.

Valle, Soledad. Trabajadores sanos y bien cuidados, 25 abril 2008.

http://www.expansionyempleo.com/edicion/ expansionyempleo/desarrollo_profesional/ salud_en_el_trabajo/es/desarrollo/1116353. html

5. En servicios de documentación para profesionales de la salud destacan portales como http:// www.uptodate.com o http://www.docguide.com; y para público general el portal más importante es http://medlineplus.gov/spanish/

6. Un ejemplo de servicio de segunda opinión médica es el de la Cleveland Clinic en Estados Unidos:

http://www.eclevelandclinic.org/productHome. jsp? productId=standard. Respecto a los portales de autogestión de la salud, merece especial atención el desarrollado por Google y Microsoft: https://www.google.com/health

7. El mundo. 160 millones de "cibercondríacos" en EUA, 8 agosto 2007.

http://www.elmundo.es/elmundosalud/2007/ 08/07/medicina/1186498932.html

8. Esta iniciativa ha sido adoptada por varios hospitales, entre ellos el Hospital Ramón y Cajal que envía a sus pacientes mensajes de texto para recordarles las citas con el médico.

El país. El hospital Ramón y Cajal recuerda las citas de consultas con mensajes SMS, 26 abril 2007.

http://www.elpais.com/articulo/red/ Hospital/Ramon/Cajal/recuerda/citas/ consultas/mensajes/SMS/elpeputeccib/ 20070426elpcibenr_1/Tes

9. El teléfono móvil se ha convertido en el sistema más utilizado por los hospitales para comunicarse con los pacientes, ya que evita desplazamientos al enfermo, ahorra costes sanitarios e incluso ayuda a reducir las listas de espera. Internet ha sido relegado a un segundo plano como medio de comunicación entre el personal médico y el paciente.

Corisco, María. La telefonía móvil desbanca a internet como sistema de comunicación con el paciente, 5 enero 2008.

http://www.elmundo.es/suplementos/salud/ 2008/738/1199487603.html

10. Todos los infográficos realizados por la Clí nica Universidad de Navarra pueden ser consultados en:

http://www.cun.es/nc/multimedia/historicoinfograficos-y-animaciones/

11. Colle (2004) considera que hay tres estilos de infográficos: a) científicos o técnicos, b) de divulgación y c) periodísticos o noticiosos.

\section{Bibliografía}

Baldwin, Line; Clarke, Malcolm; Eldabi, Tillal; Jones, Russel. "Telemedicine and its role in improving communication in healthcare". Logistic information management, 2002, v. $15, \mathrm{n}$. 4, pp. 309-319.

Barnes, Stuart; Scornavacca, Eusebio. "Mobile marketing: the role of permission and acceptance". Intl Journal of Mobile Communications, 2004, v. 2, n. 2, pp. 128-139.

Bensing, Jozien; Verheul, W.; Van Dulmen, A M. "Patient anxiety in the medical encounter: A study of verbal and nonverbal communication in general practice". Health education, 2008, v 108, n. 5, pp. 373-383.

Berry, Diane. Health communication: theory and practice. Maidenhead: Open University Press, 2007, ISBN: 0335218717.

Brashers, Dale; Babrow, Austin. "Theorizing health communication". Communication Studies, 1996, v. 47, pp. 243-251.

Byrne, Zinta. "Different media for organizational communication: Perceptions of quality and satisfaction". Journal of business and psychology, 2006, v. 21, n. 2, pp. 149-173.

Colle, Raymond. "Infografía: tipología”. Revista latina de comunicación social, 2004, v. 58. http://www.ull.es/publicaciones/latina/latina_ art660.pdf.

Fisac, Vicente. "La comunicación de los avances terapéuticos: principios éticos y responsabilidad del periodista”. En: De Quiroga, Santiago (ed). Comunicar es salud. Madrid: Prentice Hall. Financial Times, 2006, pp. 39-40. IZAN: 8483220849 .

Franco-Álvarez, Guillermina. La infografía periodística. Las Palmas de Gran Canaria: Anroart, 2005, IZAN: 8493458228.

Gosselin, Pierre. "Use of an internet 'viral' marketing software platform in health promotion". Journal of medical internet research, 2006, v. 10, n. 4.

Guttman, Nurit. "Ethics in health communication interventions". En: Thompson, Teresa; Dorsey, Alicia; Miller, Katherine y Parrott, Roxanne (Eds.). Handbook of health communication. Mahwah, N. J.: Lawrence Erlbaum Assoc, 2003, pp. 651-679. ISBN: 0805838570.

Gwyn, Richard. Communicating health and illness. London: Sage, 2002, ISBN: 0761964754.

Hornik, Robert. Public health communication: evidence for behaviour change. Mahwah, N. J.: Lawrence Erlbaum Assoc, 2002, ISBN: 0805831762 .

Houston, Thomas; Allison, Jeroan. "Users of internet health information: Differences by health status". Journal of medical internet research, 2002, v. 4, n. 2, pp. 7.

Jackson, Nigel. "Escaping from the straitjacket: UK MPs and their newsletters". Aslib proceedings, 2004, v. 56, n. 6, pp. 335-343.

Lallana, Fernando. "Diseño y color infográfico". Revista latina de comunicación social, 1999 , v. 13

http://www.ull.es/publicaciones/latina/a1999c/ 150lallana.htm.

Long, Mary; McMellon, Charles; Clark, Sylvia; Schiffman, Leon. "Building relationships with business and leisure flyers. Perceived loyalty and frequent flyer programs". Services marketing quarterly, 2006, v. 28, n. 1, pp. 1-17.

Manovich, Lev. El lenguaje en los nuevos medios de comunicación: la imagen en la era digital (trad. Fontrodona, O.). Barcelona: Paidós, 2005, ISBN: 844931769x.

Martínez-Solana, Yolanda. "El periodista de salud del siglo XXI". En: De Quiroga, Santiago (ed). Comunicar es salud. Madrid: Prentice Hall. Financial times, 2006, pp. 99-112. ISBN: 8483220849 .

Perey, Christine. "Mobile social networking: the brand at play in the circle of friends with mobile communities representing a strong opportunity for brands". International journal of mobile marketing, 2008, v. 3, n. 2, pp. 75-79.

Reid, Graham. "Building an accreditation scheme for health and social care information". Health info internet, 2008, v. 61, pp. 3-5.

Reuther, Phyllis. "Mobile search work for the mobile ecosystem: implications for operatos, portals, advertisers, and brands". Intl journal of mobile marketing, 2008, v. 3, n. 2, pp. 25-33. 
Scharl, Arno; Dickinger, Astrid; Murphy, Jamie. "Diffusion and success factors of mobile marketing". Electronic commerce research and applications, 2005, v. 4, pp. 159-173.

Sierra-Caballero, Francisco. Comunicación, educación y desarrollo: apuntes para una historia de la comunicación educativa. Sevilla: Comunicación social, 2002, ISBN: 8493225126.

Spyt, Thomas; Watt, Pamela; Boehm Maria Stafford, Paul. "Online patient support systems - is there a need?" British Journal of Clinical Governance, 2002, v. 7 n.4, pp. 250-254

Suárez-Riveiro, José-Manuel; Fernández-Suárez, Ana-Patricia. El aprendizaje autorregulado: variables estratégicas, motivacionales, evaluación e intervención. Madrid: UNED, 2004, ISBN: 843623331X.

Turner, Jeanine-Warisse. "Telemedicine: expanding health care into virtual environments". En: Thompson, Teresa; Dorsey, Alicia; Miller,
Katherine y Parrott, Roxanne (Eds.). Handbook of health communication. Mahwah, N. J.: Lawrence Erlbaum Assoc., 2003, pp. 527-528. ISBN: 0805838570 .

Valero-Sancho, José-Luis. "Visualidad del producto gráfico". Revista latina de comunicación social, 2002, v. 51.

http://www.ull.es/publicaciones/latina/ 2002junio5106valero.htm

Valero-Sancho, José-Luis. La infografía: técnicas, análisis y usos periodísticos. Barcelona: Univ Autónoma de Barcelona, 2001, ISBN: 8437049369.

Valero-Sancho, José-Luis. "La infografía en prensa". Revista latina de comunicación social, 2000, v. 30.

http://www.ull.es/publicaciones/latina/ aa2000qjn/99valero.htm

Wright, Kevin; Sparks, Lisa; O'hair, Dan. Health communication in the 21st cen- tury. Malden: Blackwell Pub., 2008, ISBN: 9781405155939

Toni González-Pacanowski, Facultad de Ciencias Sociales, Universidad Abat Oliba

Bellesguard, 30

08022 Barcelona, España.

tonigonza@telefonica.net

Pablo Medina-Aguerrebere, Departamento de Empresa Informativa, Facultad de Comunicación Campus Universidad de Navarra 31080 Pamplona, España. pamedina@unav.es

\section{conservación-restauración}
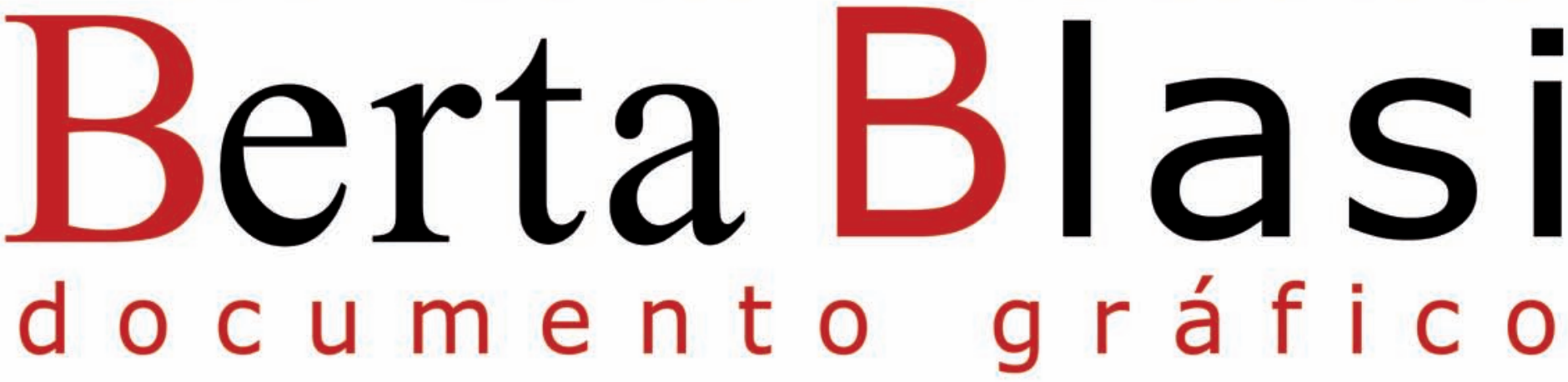

Conservación y restauración de papel, piel, pergamino y otros soportes.

Conservación y restauración de legajos y encuadernaciones.

Encuadernción artesanal personalizada.

Asesoría sobre almacenaje, conservación preventiva y preservación digital para instituciones, archivos, bibliotecas, museos, galerías, particulares...

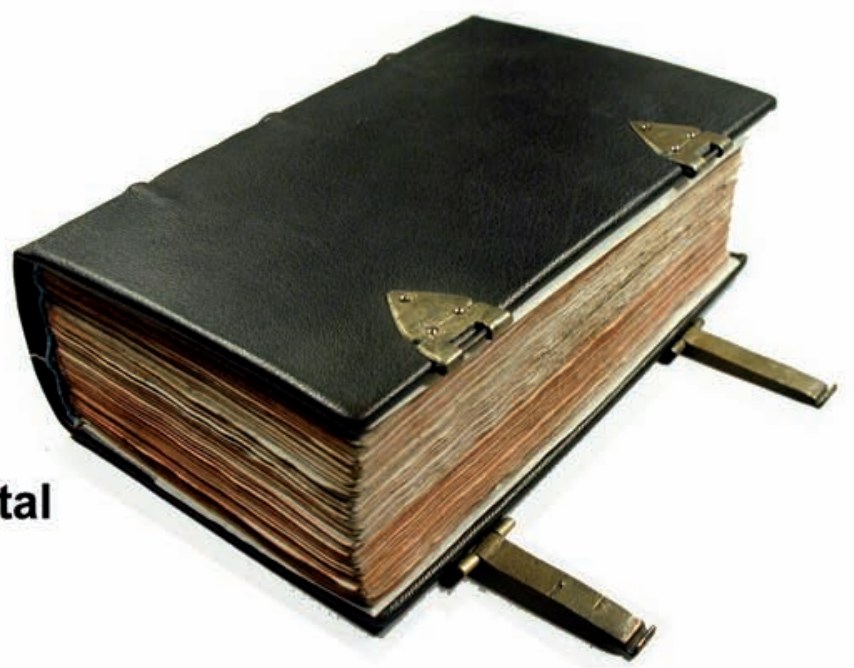

taller@bertablasi.com | wWW.bertablasi.com | (+34) 626266293 